\title{
Endoscopic reinforcement of the anastomosis followed by targeted endoscopic hemostasis for massive upper gastrointestinal bleeding after Whipple's surgery
}

Although it occurs infrequently in patients undergoing the procedure, upper gastrointestinal (GI) bleeding after Whipple's gastropancreaticoduodenectomy can be a devastating complication. Early GI bleeding that occurs within 24 to 48 hours postoperatively is an important cause of morbidity and mortality [1]. Emergency endoscopic procedures performed within a short time after major surgery have tra- ditionally been discouraged on the grounds that insufflation may disrupt the anastomotic site and lead to perforation. Therefore, some experts use interventional radiology with embolization [2]. Herein, we present a novel endoscopic approach and solution to the management of a patient with renal failure and a contraindication to interventional radiology after Whipple's surgery.
On the first day after undergoing a Whipple's gastropancreaticoduodenectomy to resect a tumor of the pancreatic head, a 53-year-old woman developed massive hematemesis and a sudden drop in her hemoglobin level from 9.6 to $5.6 \mathrm{~g} / \mathrm{dL}$, with associated hypotension and tachycardia. Because her creatinine level was $1.9 \mathrm{mg} / \mathrm{dL}$, the administration of intravenous contrast and thus interventional radiology were contraindicated.

An emergency esophagogastroduodenoscopy (EGD), performed with minimal carbon dioxide (instead of air) insufflation, showed the stomach to be filled with clots and fresh blood ( Fig.1a, Video 1). After the stomach had been partially cleared of blood and clots with an overtube (Guardus; US Endoscopy, Mentor, Ohio, USA) ( Video 1 ), active bleeding was

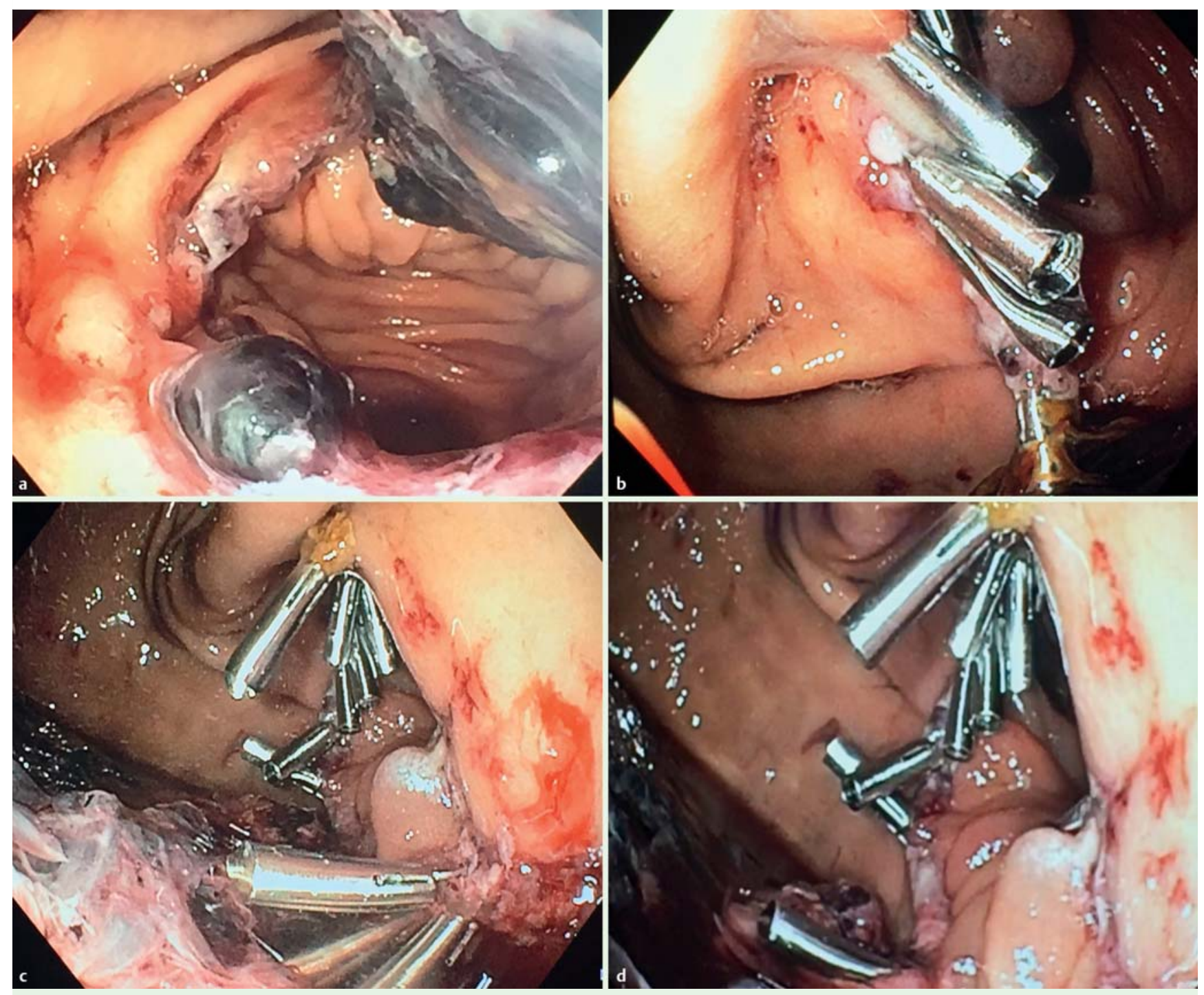

Fig. 1 Endoscopic management of massive upper gastrointestinal bleeding in a 53-year-old woman following Whipple's gastropancreaticoduodenectomy to resect a tumor of the pancreatic head. a Anastomosis with dark clot after exhaustive washing. b Deployment of clips at the distal anastomosis. $\mathbf{c}$ Sequence of clips for reinforcement at the proximal anastomosis. $\mathbf{d}$ Final aspect of the anastomosis reinforced with clips and targeted clipping of the bleeding lesion at the gastric side of the anastomosis. 


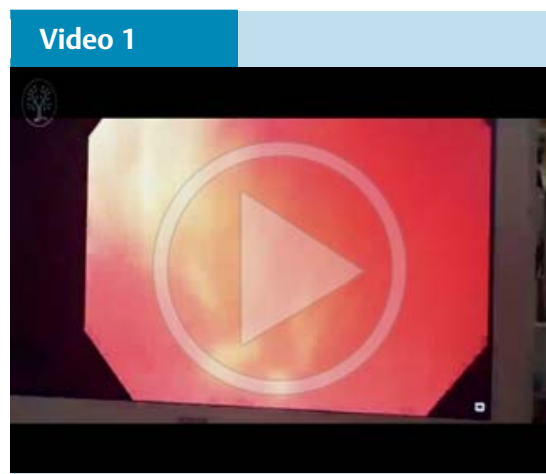

Endoscopic reinforcement of the anastomosis followed by targeted endoscopic hemostasis for massive upper gastrointestinal bleeding after Whipple's surgery.

identified at multiple points at the anastomosis. To prevent anastomotic dehiscence, the entire anastomotic line was carefully reinforced with a total of 10 clips (Cook Medical, Winston-Salem, North Carolina, USA) ( Fig. 1 b, $\bullet$ Video 1 ). Once the anastomosis had been secured, further lavage and removal of blood and blood clots were carried out. At that time, the major sites of bleeding were clearly identified and targeted with four additional clips ( $\bullet$ Fig.1c), upon which the hemorrhage ceased $(\bullet$ Fig. 1 d, $\bullet$ Video 1 ). The patient remained stable throughout the procedure, and no further episodes of bleeding occurred. Feeding was started at 16 hours after the procedure, and she was discharged home in stable condition 36 hours later.

This case is of interest for several reasons. First, we show that the careful use of minimal insufflation with carbon dioxide does not disrupt the anastomotic site. Second, the use of an overtube can facilitate the removal of blood clots and fresh blood and therefore the identification of the surgical anatomy and potential sites of bleeding (duodenojejunostomy, hepaticojejunostomy, or pancreaticojejunostomy). Finally, the novel concept of reinforcing the anastomosis allowed further endoscopic inspection, and subsequent definitive endoscopic hemostasis spared the patient surgical reintervention, with its associated morbidity and mortality.

Endoscopy_UCTN_Code_TTT_1AO_2AD

Competing interests: None

Marco A. D’Assunçaoo ${ }^{1,2}$,

Paul T. Kröner ${ }^{1,3}$, Ujjwal Kumar ${ }^{1}$, Juan P. Gutierrez ${ }^{1}$, Lucia C. Fry ${ }^{1}$, Klaus Mönkemüller ${ }^{1}$

${ }^{1}$ Basil I. Hirschowitz Endoscopic Center of Excellence, Division of Gastroenterology and Hepatology, University of Alabama at Birmingham, Birmingham, Alabama, USA
2 Digestive Endoscopy Unit, Hospital Sírio-Libanês, São Paulo, Brazil

${ }^{3}$ Department of Internal Medicine, Mount Sinai St. Luke's and Mount Sinai Roosevelt, New York, New York, USA

\section{References}

1 Wente MN, Shrikhande SV, Kleeff J et al. Management of early hemorrhage from pancreatic anastomoses after pancreaticoduodenectomy. Dig Surg 2006; 23: 203-208

2 Darnis B, Lebeau R, Chopin-Laly X et al. Postpancreatectomy hemorrhage ( $\mathrm{PPH})$ : predictors and management from a prospective database. Langenbecks Arch Surg 2013; 398: $441-448$

\section{Bibliography}

Dol http://dx.doi.org/

10.1055/s-0041-110592

Endoscopy 2016; 48: E22-E23

(c) Georg Thieme Verlag KG

Stuttgart · New York

ISSN 0013-726X

Corresponding author Klaus Mönkemüller, MD, PhD, FASGE

Division of Gastroenterology and Hepatology Basil Hirschowitz Endoscopic Center of Excellence Endoscopy Unit, JT 664

619 19th Street $S$

Birmingham, AL 35249

USA

Fax: +1-205-297-9411

klaus1@uab.edu 